\title{
Differentiating Colorectal Carcinoma From Diverticulitis With Computerised Tomography: Does Every Patient Need Follow-Up Colonoscopy After an Episode of Acute Diverticulitis?
}

Sandeep Singh ${ }^{1}$, James Shuttleworth ${ }^{1}$, Upekha Alagoda ${ }^{1}$, Alice Giucca ${ }^{1}$, Anna Heylen ${ }^{1}$, Nick Browning ${ }^{1}$ , Haytham Sumrien ${ }^{1}$

1. General and Colorectal Surgery, North Bristol NHS Trust, Bristol, GBR

Corresponding author: Sandeep Singh, dr.singhsandeep@yahoo.com

\section{Abstract}

\section{Purpose}

To correlate computerised tomography (CT) and endoscopic follow-up (FU) in differentiating presentations of acute diverticulitis (AD) and colorectal carcinoma (CRC).

\section{Methods}

Patient's discharge summaries between April 2018 and September 2019, stating AD under-diagnosis were retrieved. Admission details, CT reports, endoscopic findings and histopathology results were retrospectively collected from prospectively maintained data.

\section{Results}

In our study period of 17 months, we identified 150 patients with an admission diagnosis of AD. In total, 134 patients had a CT confirmed diagnosis of AD; 61\% had uncomplicated acute diverticulitis (UAD) and 39\% complicated acute diverticulitis (CAD). The mean age of the patients was 64 years, and $59 \%$ were female. Of the 134,15 patients were excluded, and 119 with AD were discharged with a plan to have FU endoscopy. Overall, $75 \%$ of the patients managed to undergo complete endoscopic investigation, $4 \%$ had incomplete endoscopy, and 21\% failed to attend endoscopy. Follow-up (FU) endoscopic investigation found polyps in 20 patients; seven were reported as tubular adenomas with low-grade dysplasia and 10 as non-concerning hyperplastic or sessile polyps. One was inflammatory, and two were malignant. CT scans for two patients with malignant polyps were reported as CAD with suspicion of sigmoid cancer in one and right-sided perforated diverticulitis in the other. Both patients were female aged over 60 years.

\section{Conclusions}

Review began $11 / 26 / 2020$ Review ended 12/10/2020 Published 12/11/2020

\section{() Copyright 2020}

Singh et al. This is an open access article distributed under the terms of the Creative Commons Attribution License CC-BY 4.0., which permits unrestricted use, distribution, and reproduction in any medium, provided the original author and source are credited.

This study demonstrates that a selective approach is more appropriate for endoscopic FU after an episode of AD. Risk stratification is required to allocate FU endoscopic investigation for patients at high risk for CRC.

Categories: General Surgery

Keywords: diverticulitis, carcinoma, ct, endoscopy, polyp

\section{Introduction}

In the United Kingdom, $50 \%$ of the population of 50 years is affected by diverticulosis; the prevalence increases with age [1]. The majority of those affected remain asymptomatic, though a proportion suffers acute diverticulitis (AD) which is described as an inflammation of diverticula, secondary to faecalith obstruction with localised mucosal inflammation [2]. AD is challenging to diagnose with acumen alone accurately. A combination of symptoms, inflammatory markers, and response to treatment guide suspicion, though ultrasound and computerised tomography (CT) are superior for diagnostic purposes [3]. CT imaging of the abdomen and pelvis is the gold standard in radiological diagnosis due to its high sensitivity and specificity $[4,5]$.

Despite clinical presentation and a suggestive CT, the occasional lack of specific symptoms, examination and radiological findings prevent diagnostic confirmation of $\mathrm{AD}$. Radiological findings such as vascular engorgement or mesenteric root fluid have been shown to have high specificity but low sensitivity for sigmoid diverticulitis [6]. Similarly, sections of colonic inflammation of $>10 \mathrm{~cm}$ are considered more specific for diverticulitis, whereas sections of $<5 \mathrm{~cm}$ or peri-colonic lymphadenopathy are more concerning for underlying carcinoma [7]. However, inflammatory bowel disease, infective colitis or malignancy may cause radiological changes similar to those observed in diverticulitis; inflammatory fat stranding, mural thickening or colonic obstruction [8]. The Royal College of Surgeons (RCS) recommended in 2014 that all 


\section{Cureus}

patients require investigation of the colonic lumen by endoscopy, barium enema or CT colonography (CTC) after an acute episode has resolved, which is typically performed after 6-8 weeks [9]. This stance is supported by the Association of Coloproctology in Great Britain and Ireland (ACPGBI) who similarly state "Barium enema or colonoscopy after the resolution of the acute episode is required to rule out another diagnosis” [10].

In the last decade, with the increasing diagnostic accuracy of CT, the necessity of follow up (FU) endoscopy to exclude colorectal carcinoma (CRC) has been questioned with no clear guidelines. We conducted this retrospective study to build upon previous work and assess whether CT findings alone are enough to diagnose $\mathrm{AD}$ and accurately exclude CRC.

\section{Materials And Methods}

This retrospective study was conducted over 17 months (25/04/2018 to 25/09/2019) at our unit. Sequential surgical records of patients treated for acute diverticulitis were reviewed. Patients were identified by confirmed diagnoses or diagnostic suspicion of diverticulitis from discharge summaries stored in our hospital's secured database, Integrated Clinical Environment (ICE).

Admission details, CT reports, endoscopy findings and histopathology results were retrospectively collected from prospectively maintained data. CT reports from Synapse (Picture Archiving and Communication System - PACS) were coordinated with admissions, and assessment of diagnostic confidence was made. Radiology consultants reported all scans.

CT scan requests were correlated with Endoweb, an endoscopy reporting software. Cross-sectional imaging was compared with direct visualisation to evaluate the benefits and deficiencies of both modalities in the diagnosis of AD and CRC. Subjects who deceased before discharge or during follow-up and who underwent inpatient surgical intervention like Hartmann's procedure were excluded from FU, as were those where who were too frail for invasive intervention. A further group of patients who had a recent endoscopy or CTC were not included in our study.

The study was carried out within the framework of our institute, and the protocol was approved by The Patient Safety, Assurance and Audit Service (PSAAS) committee of North Bristol NHS Trust to proceed as an observational study. Informed consent was waived, and no identifying information (such as names, images and identifying data) is included in the article.

\section{Statistical Analysis}

For the descriptive analysis, mean was used for normally distributed data. For comparing numerical data, an unpaired t-test was used when dealing with normally distributed data and Mann-Whitney U test for nonparametric data.

\section{Results}

We identified 150 patients who were diagnosed with $\mathrm{AD}$ during our study period of 17 months. Interestingly, 40 (27\%) were known to have a diverticular disease (DD). CT scan was performed for 145 of them to confirm the diagnosis of $\mathrm{AD}$, and five patients were diagnosed clinically without CT. Table 1 highlights the findings of CT scans.

\begin{tabular}{|l|l|}
\hline CT Findings & Number of patients \\
\hline UAD & $82(57 \%)$ \\
CAD & $52(35 \%)$ \\
Colitis & $6(4 \%)$ \\
Colon Cancer & $1(1 \%)$ \\
Diverticulosis only & $3(2 \%)$ \\
Normal CT & $1(1 \%)$ \\
\hline
\end{tabular}

TABLE 1: Findings of CT scan $(n=145)$

CT- computerised tomography; UAD- uncomplicated acute diverticulitis; CAD- complicated acute diverticulitis; 


\section{Cureus}

In total, 134 patients had a CT confirmed diagnosis of AD; 61\% had uncomplicated acute diverticulitis (UAD) and $39 \%$ complicated acute diverticulitis (CAD). The mean age of the patients was 64 years. The ratio of male to female patients was $41 \%$ and $59 \%$.

Of the 134, 15 were excluded because of frailty, emergency surgical intervention like Hartmann's procedure, inpatient (IP) endoscopy, recent endoscopic evidence of diverticulosis and mortality (Table 2). In total, 119 patients with AD were discharged with a plan to have FU endoscopy. Several patients who underwent complete endoscopic investigation in the form of either flexible sigmoidoscopy or colonoscopy were 89 (75\%), 5 (4\%) had incomplete endoscopy due to poor bowel preparation or intolerance, and 25 (21\%) failed to attend endoscopy (Figure 1 \& 2).

\begin{tabular}{|l|l}
\hline Reason for Exclusion & Number of patients \\
\hline Deceased (Inpatient) & 2 \\
\hline Deceased after discharge- grade 3 subarachnoid haemorrhage from an internal carotid aneurysm & 1 \\
Hartmann's procedure & 3 (2 as inpatient, 1 elective) \\
Frail- Not fit for any intervention or investigation & 6 \\
IP flexible sigmoidoscopy & 3
\end{tabular}

TABLE 2: Excluded patients $(n=15)$

Patients diagnosed with $\mathrm{AD}$ on admission $=150$

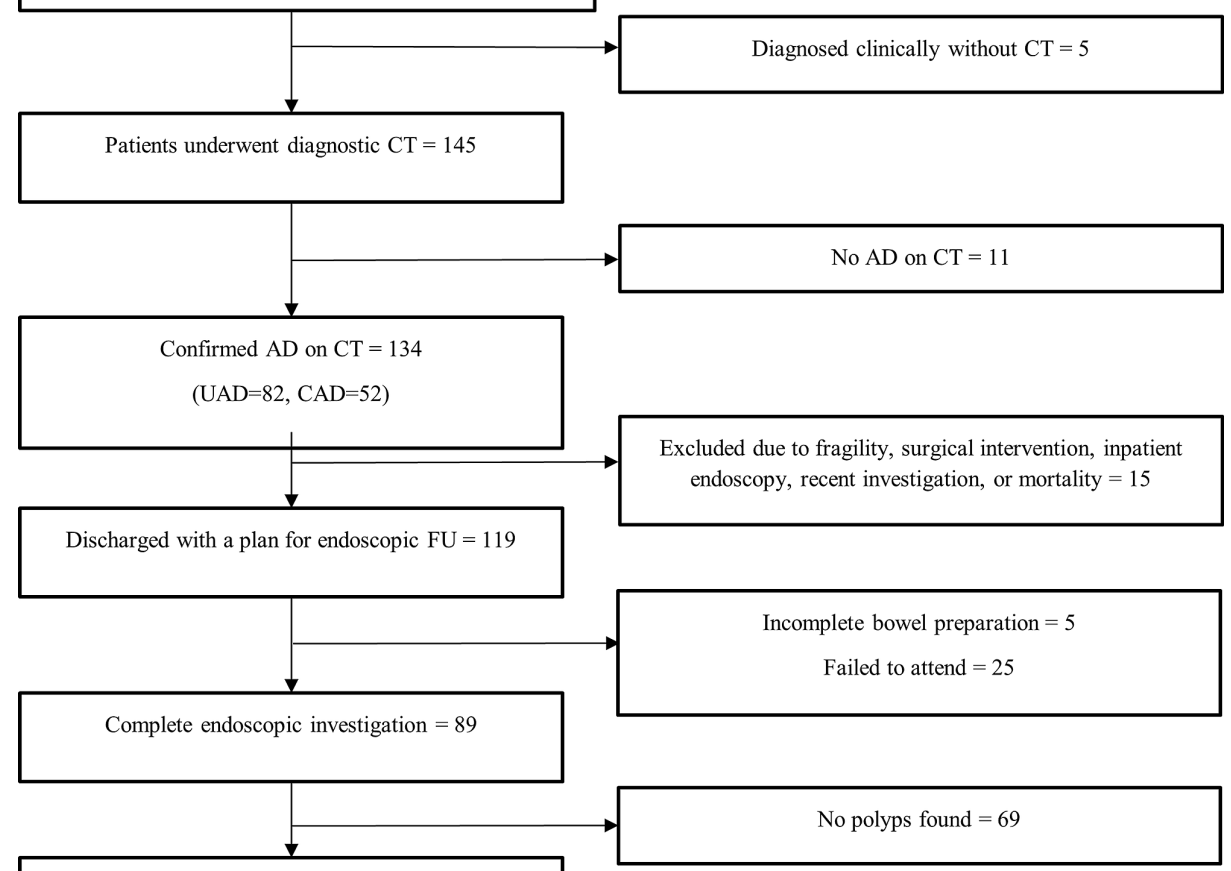

Polyps found $=20$

- $\quad$ Tubular adenomas with low grade dysplasia $=7$

- $\quad$ Non concerning or sessile polyps $=10$

- Inflammatory $=1$

- $\quad$ Malignant $=2$

FIGURE 1: Inclusion and exclusion flowchart

AD- Acute diverticulitis, CT- Computerised tomography, UAD- Uncomplicated acute diverticulitis, CADComplicated acute diverticulitis 


\section{Cureus}

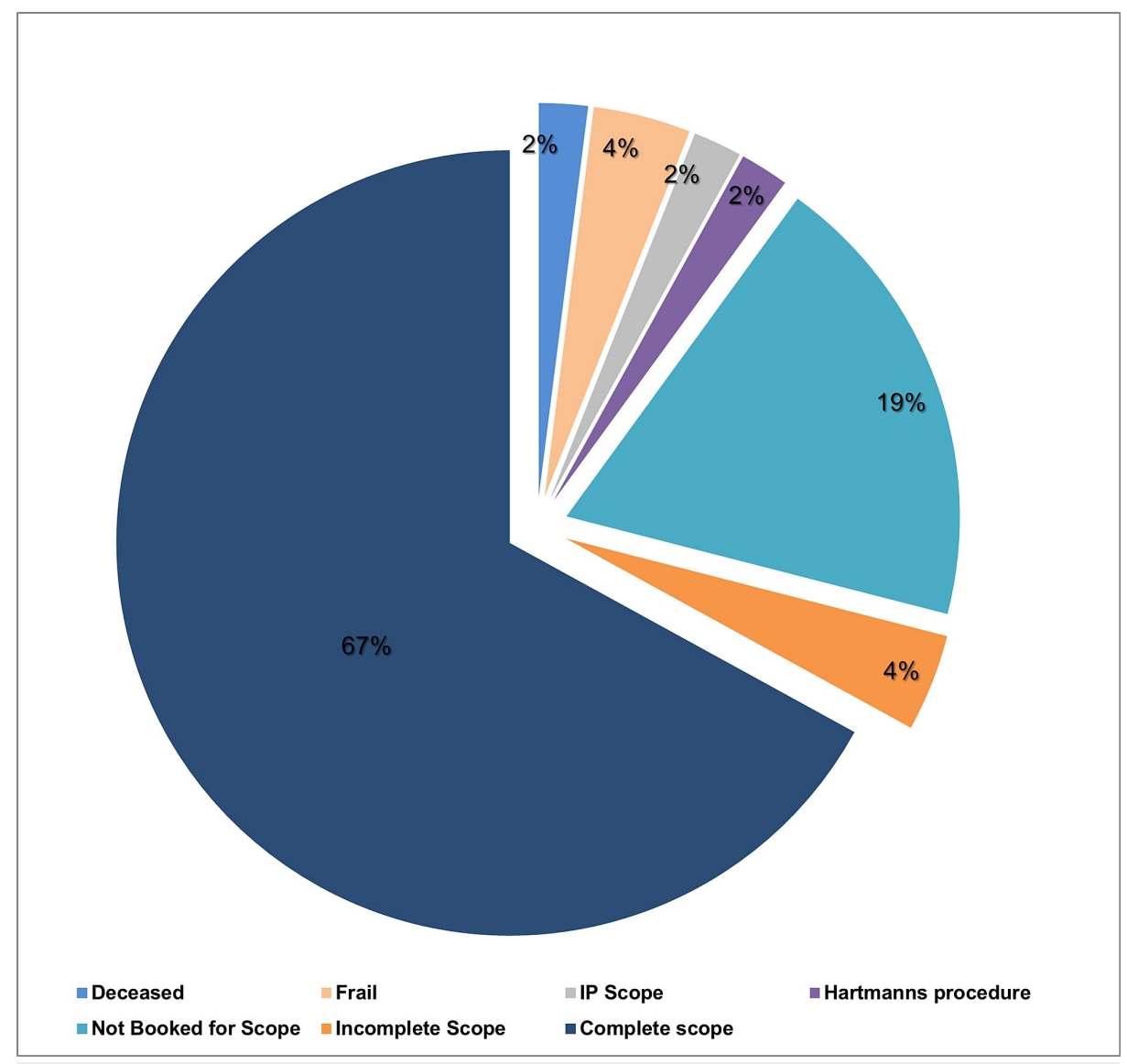

FIGURE 2: Outcome of CT proven acute diverticulitis $(n=134)$

FU endoscopic investigation found that 20 patients with polyps; 7 (9\%) were reported as tubular adenomas with low-grade dysplasia and $10(11 \%)$ as non-concerning hyperplastic or sessile polyps. One was inflammatory, and 2 (2\%) were malignant (Table 3 \& 4). Interestingly, CT scan for two patients with malignant polyps was reported as CAD with suspicion of sigmoid cancer in one and right-sided perforated diverticulitis in other (Table 5).

\begin{tabular}{|l|l|}
\hline Endoscopic findings & Number of patients \\
\hline Normal & $9(10 \%)$ \\
Mild sigmoid inflammation/ patches of erythema & $4(4 \%)$ \\
Diverticular disease & $56(63 \%)$ \\
Polyp & $20(23 \%)$ \\
\hline
\end{tabular}

TABLE 3: Endoscopy findings $(n=89)$ 


\section{Cureus}

\begin{tabular}{|c|c|c|c|c|c|}
\hline Polyps $(n=20)$ & Ascending/Caecum & Transverse & Descending & Sigmoid & Rectum \\
\hline TA $(n=7)$ & & & 2 (2 UAD) & 3 (1 UAD) (2 CAD) & 2 (2 CAD) \\
\hline$S S(n=2)$ & & 1 (UAD) & & 1 (CAD) & \\
\hline $\mathrm{HP}(\mathrm{n}=8)$ & & & 1 (CAD) & 4 (1 UAD) (3 CAD) & 3 (3 UAD) \\
\hline Inflammatory $(\mathrm{n}=1)$ & & & & 1 (CAD) & \\
\hline Malignant $(n=2)$ & 1 (CAD) & & & 1 (CAD) & \\
\hline
\end{tabular}

TABLE 4: Polyps at FU Endoscopic investigation $(n=20)$

$\mathrm{TA}=$ Tubular adenoma, $\mathrm{TV} / \mathrm{NA}=$ Tubulo-villous adenoma, $\mathrm{SS}=\mathrm{Sessile}$ serrated, HP= Hyperplastic polyp, UAD= Uncomplicated acute diverticulitis, $\mathrm{CAD}=$ Complicated acute diverticulitis

\section{Discussion}

CT scan is a widely used tool to assist diagnosis of $\mathrm{AD}$, but radiological features of $\mathrm{AD}$ and $\mathrm{CRC}$ could be incorrectly interpreted. Despite certain findings being more specific to $\mathrm{AD}$, there is overlap with features seen in CRC. Traditionally, FU colonoscopy is recommended by different colorectal societies after AD to rule out underlying malignancy.

However, in the last decade, there has been a substantive improvement in image quality and diagnostic accuracy of CT scans [11], questioning whether FU endoscopic investigation is required in patients with AD. The main aim of this study was to determine whether FU endoscopy for all patients with CT proven AD adds clinical value in ruling out an alternative diagnosis, particularly CRC, or should it be reserved for a selected subgroup of patients alone. Furthermore, a reduction in procedures would be cost-effective for NHS and less distressing and time consuming for patients.

In our study, CT scan reports were mostly concordant with FU endoscopic findings. On luminal evaluation, $80 \%$ of the patients had a diverticular disease, and $2 \%$ (two patients) were found to have a malignant polyp. Scans of both patients with latterly proven malignant changes were reported as CAD, with suspicion of sigmoid cancer in one and right-sided perforated diverticulitis in the other, which independently warrant further investigation. $\mathrm{AD}$, either uncomplicated or complicated, is not considered an independent risk factor for CRC. However, recent studies have observed a significantly higher incidence of colorectal carcinoma, particularly though not exclusively in CAD versus the general population [12].

Among the 89 patients who successfully underwent FU luminal evaluation, malignancy was diagnosed in two patients. The overall prevalence of malignancy among patients with $\mathrm{CT}$ proven $\mathrm{AD}$ was $1.5 \%$. In literature, there are many systematic reviews and meta-analyses which have demonstrated similar findings to ours and concluded that routine colonoscopy after an episode of AD is not necessary [13-17]. In these studies, the prevalence of $\mathrm{CRC}$ among patients with radiologically diagnosed $\mathrm{AD}$ was less than $2 \%$. However, few of these studies excluded patients with CAD. Our results showed that CRC prevalence was found to be higher in patients with $\mathrm{CAD}$, thus making a point that only $\mathrm{CAD}$ requires $\mathrm{FU}$ endoscopic assessment. Also, both patients with malignant polyps were female with age $>60$ years (Table 5). 


\section{Cureus}

\begin{tabular}{|c|c|c|c|c|c|c|c|}
\hline $\begin{array}{l}\text { Age Groups } \\
(n=89)\end{array}$ & \multicolumn{2}{|c|}{$\begin{array}{l}\text { Sex Female =47 } \\
\text { Male }=42\end{array}$} & $\begin{array}{l}\text { Known DD } \\
(n=17)\end{array}$ & $\begin{array}{l}\text { CT UAD } \\
(n=53)\end{array}$ & $\begin{array}{l}\text { CT CAD } \\
(n=36)\end{array}$ & $\begin{array}{l}\text { Endoscopic Findings = } \\
\text { Colonic polyp }(n=20)\end{array}$ & $\begin{array}{l}\text { Histopathology= confirmed } \\
\text { neoplasia }(n=2)\end{array}$ \\
\hline \multirow{2}{*}{$\begin{array}{l}31-50 \text { years } \\
(n=17)\end{array}$} & $F$ & 5 & 0 & 5 & 0 & 0 & 0 \\
\hline & $\mathrm{M}$ & 12 & 3 & 6 & 6 & 4 & 0 \\
\hline \multirow{2}{*}{$\begin{array}{l}51-65 \text { years } \\
(n=37)\end{array}$} & $F$ & 17 & 2 & 12 & 5 & 4 & 2 \\
\hline & $\mathrm{M}$ & 20 & 2 & 8 & 12 & 8 & 0 \\
\hline \multirow{2}{*}{$\begin{array}{l}66-80 \text { years } \\
(n=25)\end{array}$} & $F$ & 17 & 5 & 11 & 6 & 3 & 0 \\
\hline & M & 8 & 1 & 5 & 3 & 1 & 0 \\
\hline \multirow{2}{*}{$\begin{array}{l}>80 \text { years } \\
(\mathrm{n}=10)\end{array}$} & $F$ & 8 & 3 & 5 & 3 & 0 & 0 \\
\hline & $\mathrm{M}$ & 2 & 1 & 1 & 1 & 0 & 0 \\
\hline
\end{tabular}

\section{TABLE 5: Comparing CT and FU polyp detection rate with age groups}

Since 2014, the year in which the guidelines regarding endoscopic FU were published, massive improvements have been made in the quality of CT scanners to provide high-resolution images that may allow a radiologist to more easily distinguish malignancy from inflammatory changes. The physical scanning process is a mature technology; however, better display programmes allow images to be viewed with greater resolution [18].

This study shows that a selective approach based on patients with certain risk factors such as CAD, female gender, and age (>60) may be beneficial to allocate resources and minimise patient distress appropriately. However, future work is required with a larger study cohort to evaluate this preliminary finding and a proposed algorithm for diagnostic accuracy and precision. Until this is the case, we recommend routine FU luminal evaluation to differentiate AD from CRC.

The strength of this study lies in its design and primary end-point question, which lays the foundation of a more extensive study in the future. Its weakness is that it is retrospective, small cohort and reliance on patients recorded data. Accordingly, we see a particular need for further prospective research on FU endoscopic investigation after an episode of $\mathrm{AD}$ to establish the selective criteria for risk stratification.

\section{Conclusions}

Routine endoscopic evaluation after an episode of CT proven AD does not appear to be necessary to differentiate $\mathrm{AD}$ from CRC. This study justifies the need to develop a risk stratification algorithm based on CT findings, gender and age. However, further research is required with a more extensive study cohort to establish selective criteria.

\section{Additional Information \\ Disclosures}

Human subjects: Consent was obtained by all participants in this study. The Patient Safety, Assurance and Audit Service (PSAAS) committee issued approval QI32622. Methods were carried out within the framework of our institute and study protocol was approved by The Patient Safety, Assurance and Audit Service (PSAAS) committee of North Bristol NHS Trust to proceed as an observational study. Informed consent was waived and no identifying information (such as names, images and identifying data) is included in the article. Animal subjects: All authors have confirmed that this study did not involve animal subjects or tissue. Conflicts of interest: In compliance with the ICMJE uniform disclosure form, all authors declare the following: Payment/services info: All authors have declared that no financial support was received from any organization for the submitted work. Financial relationships: All authors have declared that they have no financial relationships at present or within the previous three years with any organizations that might have an interest in the submitted work. Other relationships: All authors have declared that there are no other relationships or activities that could appear to have influenced the submitted work.

\section{References}

1. Hughes LE: Postmortem survey of diverticular disease of the colon: I. diverticulosis and diverticulitis . Gut. 1969, 10:336-344. 10.1136/gut.10.5.336 
2. Matrana MR, Margolin DA: Epidemiology and pathophysiology of diverticular disease. Clinics in Colon and Rectal Surgery. 2009, 22:141-146. 10.1055/s-0029-1236157

3. Toorenvliet BR, Bakker RF, Breslau PJ, Merkus JW, Hamming JF: Colonic diverticulitis: a prospective analysis of diagnostic accuracy and clinical decision-making. Colorectal Dis. 2010, 12:179-186. 10.1111/j.1463-1318.2009.01778.x

4. Cho KC, Morehouse HT, Alterman DD, Thornhill BA: Sigmoid diverticulitis: diagnostic role of CTcomparison with barium enema studies. Radiology. 1990, 176:111-115. 10.1148/radiology.176.1.2191360

5. Hachigian, M.P., Eisenstat, T.E.: Computed tomography in the initial management of acute left-sided diverticulitis. Dis Colon Rectum. 1992, 35:1123-1129. 10.1007/BF02251962

6. A M Padidar, R B Jeffrey, Jr, J F Dolph: Differentiating sigmoid diverticulitis from carcinoma on CT scans: mesenteric inflammation suggests diverticulitis. . AJR Am J Roentgenol. 1994, 163:81-83. 10.2214/ajr.163.1.8010253

7. Chintapalli KN, Chopra S, Dodd GD 3rd: Diverticulitis versus colon cancer: differentiation with helical CT findings. Radiology. 1999, 210:429-35. 10.1148/radiology.210.2.r99fe48429

8. Destigter KK, Keating DP: Imaging update: acute colonic diverticulitis. Clin Colon Rectal Surg. 2009, 22:14755. 10.1055/s-0029-1236158

9. RCS: Colinic Diverticular Disease - Commissioning Guide. The Royal College of Surgeons of England Publications. RCS (ed): RCS Publications, London; 2017. 1-13. https://www.rcseng.ac.uk/library-andpublications/rcs-publications/docs/colinic-diverticular-disease/

10. Fozard JB, Armitage NC, Jones OM: Association of Coloproctology of Great Britain and Ireland. ACPGBI position statement on elective resection for diverticulitis. Colorectal Dis. 2011, 13:1-11. 10.1007/bf03370905

11. Pelc NJ: Recent and future directions in CT imaging. Ann Biomed Eng. 2014, 42:260-8. 10.1007/s10439-0140974-Z

12. N Azhar, P Buchwald, T Schyman: Risk of colorectal cancer following CT-verified acute diverticulitis: a nationwide population-based cohort study. Colorectal Disease. 2020, 22:1406-1414. 10.1111/codi.15073

13. Page AA, Khan A, Rajaratnam S, et al.: Is follow-up endoscopy necessary after successful medical treatment of CT-proven acute diverticulitis?. Gut. 2011, 60:72. 10.1136/gut.2011.239301.146

14. Daniels L, Unlü C, de Wijkerslooth TR, et al.: Routine colonoscopy after left-sided acute uncomplicated diverticulitis: a systematic review. Gastrointest Endosc. 2014, 79:378-89. 10.1016/j.gie.2013.11.013

15. De Vries HS, Boerma D, Timmer R, et al.: Routine colonoscopy is not required in uncomplicated diverticulitis: a systematic review. Surg Endosc. 2014, 28:2039-47. 10.1007/s00464-014-3447-4

16. Sai VF, Velayos F, Neuhaus J, et al.: Colonoscopy after CT diagnosis of diverticulitis to exclude colon cancer: a systematic literature review. Radiology. 2012, 263:383-90. 10.1148/radiol.12111869

17. Sharma PV, Eglinton T, Hider P, et al.: Systematic review and meta-analysis of the role of routine colonic evaluation after radiologically confirmed acute diverticulitis. Ann Surg. 2014, 259:263-72. 10.1097/SLA.0000000000000294

18. The Royal College of Radiologists : CT Equipment, operation, Capacity and Planning in the NHS . Clinical Imagining Board. RCR (ed): Clinical Radiology, London; 2015. 1-25.

https://www.rcr.ac.uk/sites/default/files/ct_equipment_in_the_nhs_report_cib_may_2015_v2_final240615.pdf 